\title{
Space-Time Interpolants
}

\author{
Goran Frehse $^{1}$, Mirco Giacobbe ${ }^{2(\bowtie)}$, and Thomas A. Henzinger ${ }^{2}$
}

1 Univ. Grenoble Alpes, CNRS, Grenoble INP, VERIMAG, Grenoble, France

2 IST Austria, Klosterneuburg, Austria

mgiacobbe@ist.ac.at

\begin{abstract}
Reachability analysis is difficult for hybrid automata with affine differential equations, because the reach set needs to be approximated. Promising abstraction techniques usually employ interval methods or template polyhedra. Interval methods account for dense time and guarantee soundness, and there are interval-based tools that overapproximate affine flowpipes. But interval methods impose bounded and rigid shapes, which make refinement expensive and fixpoint detection difficult. Template polyhedra, on the other hand, can be adapted flexibly and can be unbounded, but sound template refinement for unbounded reachability analysis has been implemented only for systems with piecewise constant dynamics. We capitalize on the advantages of both techniques, combining interval arithmetic and template polyhedra, using the former to abstract time and the latter to abstract space. During a CEGAR loop, whenever a spurious error trajectory is found, we compute additional space constraints and split time intervals, and use these space-time interpolants to eliminate the counterexample. Space-time interpolation offers a lazy, flexible framework for increasing precision while guaranteeing soundness, both for error avoidance and fixpoint detection. To the best of out knowledge, this is the first abstraction refinement scheme for the reachability analysis over unbounded and dense time of affine hybrid systems, which is both sound and automatic. We demonstrate the effectiveness of our algorithm with several benchmark examples, which cannot be handled by other tools.
\end{abstract}

\section{Introduction}

Formal verification techniques can be used to either provide rigorous guarantees about the behaviors of a critical system, or detect instances of violating behavior if such behaviors are possible. Formal verification has become widely used in the design of software and digital hardware, but has yet to show a similar success for physical and cyber-physical systems. One of the reasons for this is a scarcity of suitable algorithmic verification tools, such as model checkers, which are formally sound, precise, and scale reasonably well. In this paper, we propose a novel verification algorithm that meets these criteria for systems with piecewise affine dynamics. The performance of the approach is illustrated experimentally on a number of benchmarks. Since systems with affine dynamics have been studied before, we first describe why the available methods and tools do not handle this

(c) The Author(s) 2018

H. Chockler and G. Weissenbacher (Eds.): CAV 2018, LNCS 10981, pp. 468-486, 2018.

https://doi.org/10.1007/978-3-319-96145-3_25 
class of systems sufficiently well, and then describe our approach and its core contributions.

Previous Approaches. The algorithmic verification of systems with continuous or discrete-continuous (hybrid) dynamics is a hard problem both in theory and practice. For piecewise constant dynamics (PCD), the continuous successor states (a.k.a. flow pipe) can be computed exactly, and the complexity is exponential in the number of variables $[17,19]$. While in principle, any dynamics can be approximated arbitrarily well by PCD systems using an approach called hybridization [20], this requires partitioning of the state space, which often leads to prohibitive computational costs. For piecewise affine dynamics (PWA), one-step successors can be computed approximately using complex set representations. However, all published approaches suffer either from a possibly exponential increase in the complexity of the set representation, or from a possibly exponential increase in the approximation error as the considered time interval increases; this will be argued in detail in Sect. 4.

In addition to these theoretical obstacles, we note the following practical obstacles for the available tools and their performance in experiments. The only available model checkers that are (i) sound (i.e., they compute provable densetime overapproximations), (ii) unbounded (i.e., they overapproximate the flowpipe for an infinite time horizon), and (iii) arbitrarily precise (i.e., they support precision refinement) are, with one exception, limited to PCD systems, namely, HyTech [18], PHAVer [13], and Lyse [7]. The tool Ariadne [6] can deal with affine dynamics and is sound, unbounded, and precise. However, Ariadne discretizes the reachable state space with a rectangular grid. This invariably leads to an exponential complexity in terms of the number of variables. Other tools that are applicable to PWA systems do not meet our criteria in that they are either not formally sound (e.g., CORA [2], SpaceEx [15]), not arbitrarily precise because of templates or particular data structures (e.g., SpaceEx, Flow* [8], CORA), or limited to bounded model checking (e.g., dReach [24], Flow*). All the above tools exhibit fatal limitations in scalability or precision on standard PWA benchmarks; they typically work only on well-chosen examples. Note that while these tools do not meet the criteria we advance in this paper, they of course have strengths in other areas handling nonlinear and nondeterministic dynamics.

Our Approach. We view iterative abstraction refinement as critical for soundness and precision management, and fixpoint detection as critical for evaluating unbounded properties. We implement, for the first time, a CEGAR (counterexample-guided abstraction refinement) scheme in combination with a fixpoint detection criterion for PWA systems. Our abstraction refinement scheme manages complexity and precision trade-offs in a flexible way by decoupling time from space: the dense timeline is partitioned into a sequence of intervals that are refined individually and lazily, by splitting intervals, to achieve the necessary precision and detect fixpoints; state sets are overapproximated using template polyhedra that are also refined individually and lazily, by adding normal directions to templates; and both refinement processes are interleaved for optimal results, while maintaining soundness with each step. A similar approach was 
recently proposed for the limited class of PCA systems [7]; this paper can be seen as an extension of the approach to the class of piecewise affine dynamics.

With each iteration of the CEGAR loop, a spurious counterexample is removed by computing a proof of infeasibility in terms of a sequence of linear constraints in space and interval constraints in time, which we call a sequence of space-time interpolants. We use linear programming to construct a suitable sequence of space-time intervals and check for fixpoints. If a fixpoint check fails, we increase the time horizon by adding new intervals. The separation of time from space gives us the flexibility to explore different refinement strategies. Finetuning the iteration of space refinement (adding template directions), time refinement (splitting intervals), and fixpoint checking (adding intervals), we find that it is generally best to prefer fewer time intervals over fewer space constraints. Based on performance evaluation, we even expand individual intervals time when this is possible without sacrificing the necessary precision for removing a counterexample.

\section{Motivating Example}

The ordinary differential equation over the variables $x$ and $y$

$$
\begin{aligned}
& \dot{x}=0.1 x-y+1.8 \\
& \dot{y}=x+0.1 y-2.2
\end{aligned}
$$

moves counterclockwise around the point $(2,2)$ in an outward spiral. We center a box $B$ (of side 0.92 ) on the same point and place a diagonal segment $S$ close to the bottom right corner of $B$, without touching it (between $(2,1)$ and $(3.5,2)$; see Fig. 1). Then, we consider the problem of proving that every trajectory starting from any point in $S$ never hits $B$. This is a time-unbounded reachability problem for a hybrid automaton with piecewise affine dynamics and two control modes. The first mode has the dynamics above (Eq. 1) and $S$ as initial region. It has a transition to a second mode, which in its turn has $B$ as invariant. The second mode is a bad mode, which all trajectories indeed avoid.

We tackle the reachability problem by abstraction refinement. In particular, we aim at automatically constructing an enclosure for the flowpipe-i.e., for the set of trajectories from $S$-which (i) avoids the bad state $B$ and (ii) covers the continuous timeline up to infinity. Figure 1 shows three abstractions that result from different strategies for refining an initial space partition (i.e., template) and time partition (i.e., sequence of time intervals). All three refinement schemes start by enclosing $S$ with an initial template polyhedron $P$, and then transforming $P$ into a sequence of abstract flowpipe sections intflow $\left.{ }^{[t}, \bar{t}\right](P)$, one for each interval $[\underline{t}, \bar{t}]$ of an initial partitioning of the unbounded timeline. The computation of new flowpipe sections stops when a fixpoint is reached,-i.e., we reach a time threshold $t^{*}$ whose flowpipe section closes a cycle with intflow ${ }^{t^{*}}(P) \subseteq P$, sufficient condition for any further flowpipe section to be contained within the union of previously computed sections. 


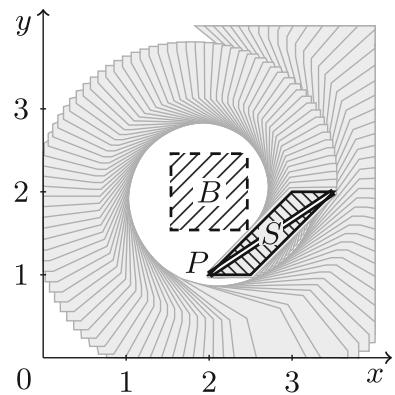

Time refinement only (a)

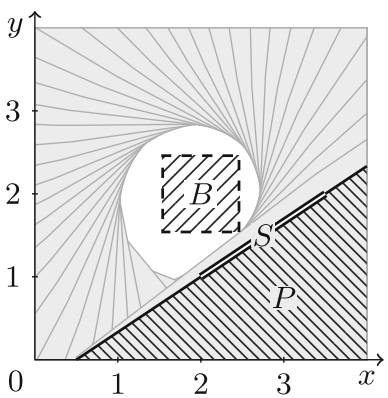

Uniform-time interpolation Ada tive-time interpolation

(b)

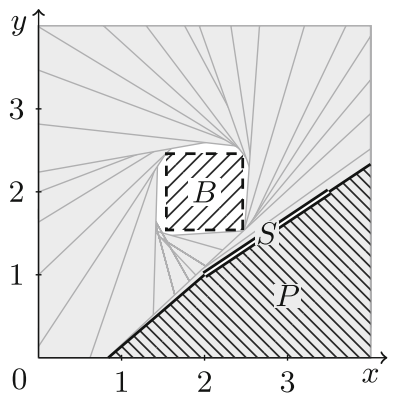

(c)

Fig. 1. Comparison of abstraction refinement methods for the ODE in Eq. 1, the segment $S$ as initial region, and the box $B$ as bad region. The polyhedron $P$ is the template polyhedron of $S$, and the gray polyhedra are the flowpipe sections intflow ${ }^{[\underline{t}, \bar{t}]}(P)$.

Refinement scheme (a) sticks to a fixed octagonal template $P$-i.e., to the normals of a regular octagon - and iteratively halves all time intervals until every flowpipe section avoids the bad set $B$. This is achieved at interval width $1 / 64$, but the computation does not terminate because no fixpoint is reached. Refinement scheme (b) splits time similarly but also computes a different, more accurate template for every iteration: first, an interval $[\underline{t}, \bar{t}]$ is halved until it admits a halfspace interpolant -i.e., a halfspace $H$ that $S \subseteq H$ and $\operatorname{intflow}^{[t, \bar{t}]}(H) \cap B=$ $\emptyset$; then, a maximal set of linearly independent directions is chosen as template from the normals of the obtained halfspaces. Refinement scheme (b) succeeds at interval width $1 / 16$ to avoid $B$ and reach a fixpoint; the latter at time 6.25 , with intflow $^{6.25}(P) \subseteq P$. Refinement scheme (c) modifies (b) by optimizing the refinement of the time partition: instead of halving time intervals, the maximal intervals which admit halfspace interpolants are chosen. This scheme produces a nonuniform time partitioning with an average interval width of about $1 / 8$, discovers five template directions, and finds a fixpoint in fewer steps.

Each iteration of the abstraction refinement loop consists of first abstracting the initial region into a template polyhedron, second solving the differential equation into a sequence of interval matrices, and finally transforming the template polyhedron using each of the interval matrices. We represent each transformation symbolically, by means of its support function. Then, we verify (i) the separation between every support function and the bad region, and (ii) the containment of any support function in the initial template polyhedron. The separation problem amounts to solving one LP, and the inclusion problem amounts to solving an LP in each template direction. If the separation fails, then we independently bisect each time that does not admit halfspace interpolants and expand each that does, until all are proven separated. Together, these halfspace interpolants form an infeasibility proof for the counterexample: a space-time interpolant. We forward the resulting new time intervals and halfspaces to the abstraction 
generator, and repeat, using the refined partitioning and the augmented template. If the inclusion fails, then we increase the time horizon by some amount $\Delta$, and repeat. Once we succeed with both separation and inclusion, the system is proved safe.

This example shows the advantage of lazily refining both the space partitioning (i.e., the template) by adding directions, and the time partitioning, by splitting intervals.

\section{$3 \quad$ Hybrid Automata with Piecewise Affine Dynamics}

A hybrid automaton with piecewise affine dynamics consists of an $n$-dimensional vector $x$ of real-valued variables and a finite directed multigraph $(V, E)$, the control graph. We call it the control graph, the vertices $v \in V$ the control modes, and the edges $e \in E$ the control switches. We decorate each mode $v \in V$ with an initial condition $Z_{v} \subseteq \mathbb{R}^{n}$, a nonnegative invariant condition $I_{v} \subseteq \mathbb{R}_{\geq 0}^{n}$, and a flow condition given by the system of ordinary differential equations

$$
\dot{x}=A_{v} x+b_{v} .
$$

We decorate each switch $e \in E$ with a guard condition $G_{e} \subseteq \mathbb{R}^{n}$ and an update condition given the difference equations $x:=R_{e} x+s_{e}$. All constraints $I, G$, and $Z$ are conjuctions of rational linear inequalities, $A$ and $R$ are constant matrices, and $b$ and $s$ constant vectors of rational coefficients. In this paper, whenever an indexing of modes and switches is clear from the context, we index the respective constraints and transformations similarly, e.g., we abbreviate $A_{v_{i}}$ with $A_{i}$.

A trajectory is a possibly infinite sequence of states $(v, x) \in V \times \mathbb{R}^{n}$ repeatedly interleaved first by a switching time $t \in \mathbb{R}_{\geq 0}$ and then by a switch $e \in E$

$$
\left(v_{0}, x_{0}\right) t_{0}\left(v_{0}, y_{0}\right) e_{0}\left(v_{1}, x_{1}\right) t_{1}\left(v_{1}, y_{1}\right) e_{1} \ldots
$$

for which there exists a sequence of solutions $\psi_{0}, \psi_{1}, \ldots: \mathbb{R} \rightarrow \mathbb{R}_{n}$ such that $\psi_{i}(0)=x_{i}, \psi_{i}\left(t_{i}\right)=y_{i}$ and they satisfy (i) the invariant conditions $\psi_{i}(t) \in I_{i}$ and (ii) the flow conditions $\dot{\psi}_{i}(t)=A_{i} \psi_{i}(t)+b_{i}$, for all $t \in\left[0, t_{i}\right]$. Moreover, $x_{0} \in Z_{0}$, every switch $e_{i}$ has source $v_{i}$, destination $v_{i+1}$, and the respective states satisfy (i) the guard condition $y_{i} \in G_{i}$ and (ii) the update $x_{i+1}=R_{i} y_{i}+s_{i}$. The maximal set of its trajectories is the semantics of the hybrid automaton, which is safe if none of them contains a special bad mode.

Every hybrid automaton with affine dynamics can be transformed into an equivalent hybrid automaton with linear dynamics, i.e., the special case where $b=0$ on every mode. We obtain such transformation by adding one extra variable $y$, rewriting the flow of every mode into $\dot{x}=A x+b y$, and forcing $y$ to be always equal to 1 , i.e., invariant $y=1$ and flow $\dot{y}=0$ on every mode and update $y^{\prime}=y$ on every switch. For this reason, in the following sections we discuss w.l.o.g. the reachability analysis of hybrid automata with linear dynamics. 


\section{Time Abstraction Using Interval Arithmetic}

We abstract the reach set of the hybrid automaton with a union of convex polyhedra. In particular, we abstract the states that are reachable in a mode using a finite sequence of images of the initial region over a time partitioning, until a completeness threshold is reached. Thereafter, we compute the template polyhedron of each of the images that can take a switch. Then, we repeat in the destination mode and we continue until a fixpoint is found.

Precisely, a time partitioning $T$ is a (possibly infinite) set of disjoint closed time intervals whose union is a single (possibly open) interval. For a finite set of directions $D \subseteq \mathbb{R}^{n}$, the $D$-polyhedron of a closed convex set $X$ is the tightest polyhedral enclosure whose facets normals are in $D$. In the following, we associate every mode $v$ to a template $D_{v}$ and a time partitioning $T_{v}$ of the time axis $\mathbb{R}_{\geq 0}$, we employ interval arithmetic for abstracting the continuous dynamics (Sect. 4.1 ), and on top of it we develop a procedure for hybrid dynamics (Sect. 4.2).

\subsection{Continuous Dynamics}

We consider w.l.o.g. a mode with ODE reduced to the linear form $\dot{x}=A_{v} x$, invariant $I_{v}$, and a given time interval $[\underline{t}, \bar{t}]$. Every linear ODE $\dot{x}=A x$ has the unique solution

$$
\psi(t)=\exp (A t) \psi(0) .
$$

It follows (see also [16]) that the set of states reachable in $v$ after exactly $t$ time units from an initial region $X$ is

$$
\text { flow }_{v}^{t}(X) \stackrel{\text { def }}{=} \exp \left(A_{v} t\right) X \cap \bigcap_{0 \leq \tau \leq t} \exp \left(A_{v}(t-\tau)\right) I_{v},
$$

Then, the flowpipe section over the time interval $[\underline{t}, \bar{t}]$ is

$$
\text { flow }_{v}^{[\underline{t}, \bar{t}]}(X) \stackrel{\text { def }}{=} \cup\left\{\text { flow }_{v}^{t}(X) \mid t \in[\underline{t}, \bar{t}]\right\} \text {. }
$$

We note three straightforward but consequential properties of the reach set: (i) The accuracy of any convex abstraction depends on the size of the time interval: While $\operatorname{flow}_{v}^{t}(X)$ is convex for convex $X$, this is generally not the case for flow ${ }_{v}^{[t, \bar{t}]}(X)$. (ii) We can prune the time interval whenever we detect that the reach set no longer overlaps with the invariant: If for any $t^{*} \geq 0$, flow $_{v}^{t^{*}}(X)=\emptyset$, then for all $\bar{t} \geq t^{*}$, flow ${ }_{v}^{\bar{t}}(X)=\emptyset$ and flow ${ }_{v}^{[t, \bar{t}]}(X)=$ flow $_{v}^{\left[\underline{t}, t^{*}\right]}(X)$. (iii) We can prune the time interval whenever we detect containment in the initial states: If flow $_{v}^{t^{*}}(X) \subseteq X$, then flow $\frac{[t, \infty]}{v}(X)=$ flow $_{v}^{\left[\underline{t}, t^{*}\right]}(X)$.

For given $A$ and $t$, the matrix $\exp (A t)$ can be computed with arbitrary, but only finite, accuracy. We resolve this problem by computing a rational interval matrix $[\underline{M}, \bar{M}]$, which we denote $\operatorname{intexp}(A, \underline{t}, \bar{t})$, such that for all $t \in[\underline{t}, \bar{t}]$ we have element-wise that

$$
\exp (A t) \in \operatorname{intexp}(A, \underline{t}, \bar{t})
$$


This interval matrix can be derived efficiently with a variety of methods [25], e.g., using a guaranteed ODE solver or using interval arithmetic. The width of the interval matrix can be made arbitrarily small at the price of increasing the number of computations and the size of the representation of the rational numbers. In our approach, we do not rely in a fixed accuracy of the interval matrix. Instead, we require that the accuracy increases as the width of the time interval goes to zero. That way, we don't need to introduce an extra parameter. To ensure progress in our refinement loop, we require that the interval matrix decreases monotonically when we split the time interval. Formally, if $[\underline{t}, \bar{t}] \subseteq[\underline{u}, \bar{u}]$ we require the element-wise inclusion $\operatorname{intexp}(A, \underline{t}, \bar{t}) \subseteq \operatorname{intexp}(A, \underline{u}, \bar{u})$. This can be ensured by intersecting the interval matrices with the original interval matrix after time splitting.

While the mapping with interval matrices is in general not convex [29], we can simplify the problem by assuming that all points of $X$ are in the positive orthant. As long as $X$ is bounded from below, this condition can be satisfied by inducing an appropriate coordinate change. Under the assumption that $X \subseteq \mathbb{R}_{\geq 0}^{n}$,

$$
[\underline{M}, \bar{M}](X)=\left\{y \in \mathbb{R}^{n} \mid \underline{M} x \leq y \leq \bar{M} x \text { and } x \in X\right\} .
$$

Combining the above results, we obtain a convex abstraction of the flowpipe over a time interval as

$$
\operatorname{intflow}_{v}^{[\underline{t}, \bar{t}]}(X) \stackrel{\text { def }}{=} \operatorname{intexp}(A, \underline{t}, \bar{t}) X \cap I_{v} .
$$

The abstraction is conservative in the sense that flow ${ }_{v}^{[t, \bar{t}}(X) \subseteq \operatorname{intflow}_{v}^{[t, \bar{t}]}(X)$. On the other hand, the longer is the time interval, the coarser is the abstraction. For this reason, we construct an abstraction of the flowpipe in terms of a union of convex approximations over a time partitioning. The abstract flowpipe over the time partitioning $T$ is

$$
\operatorname{intflow}_{v}^{T}(X) \stackrel{\text { def }}{=} \cup\left\{\operatorname{intflow}_{v}^{[t, \bar{t}]}(X) \mid[\underline{t}, \bar{t}] \in T\right\} .
$$

Again, this is conservative w.r.t. the concrete flowpipe, i.e., for all time partitionings $T$ it holds that $\operatorname{flow}_{v}^{\cup T}(X) \subseteq \operatorname{intflow}_{v}^{T}(X)$. Moreover, it is conservative w.r.t. any refinement of $T$, i.e., the time partitioning $U$ refines $T$ if $\cup U=\cup T$ and $\forall[\underline{u}, \bar{u}] \in U: \exists[\underline{t}, \bar{t}] \in T:[\underline{u}, \bar{u}] \subseteq[\underline{t}, \bar{t}]$, then $\operatorname{intflow~}_{v}^{U}(X) \subseteq \operatorname{intflow}_{v}^{T}(X)$.

\subsection{Hybrid Dynamics}

We embed the flowpipe abstraction routine into a reachability algorithm that accounts for the switching induced by the hybrid automaton. The discrete post operator is the image of a set $Y \subseteq \mathbb{R}^{n}$ through a switch $e \in E$

$$
\operatorname{jump}_{e}(Y) \stackrel{\text { def }}{=} R_{e}\left(Y \cap G_{e}\right) \oplus\left\{s_{e}\right\} .
$$

We explore the hybrid automaton constructing a set of abstract trajectories, namely sequences abstract states interleaved by time intervals and switches

$$
\left(v_{0}, X_{0}\right)\left[\underline{t}_{0}, \bar{t}_{0}\right]\left(v_{0}, Y_{0}\right) e_{0}\left(v_{1}, X_{1}\right)\left[\underline{t}_{1}, \bar{t}_{1}\right]\left(v_{1}, Y_{1}\right) e_{1} \ldots
$$




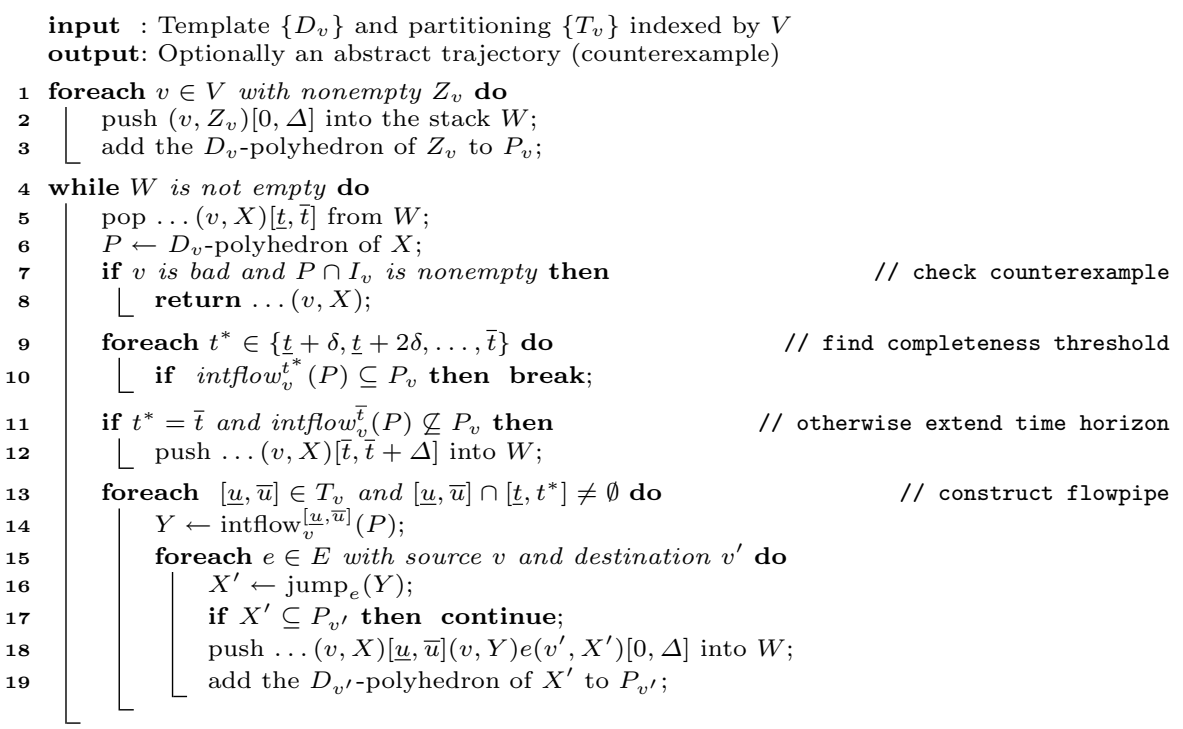

Algorithm 1. Reachability procedure.

where $X_{0}, Y_{0}, \cdots \subseteq \mathbb{R}^{n}$ are nonempty sets of states that comply with template $\left\{D_{v}\right\}$ and partitioning $\left\{T_{v}\right\}$ in the following sense. First, $X_{0}=Z_{0}$ and $X_{i+1}=\operatorname{jump}_{i}\left(Y_{i}\right)$ for all $i \geq 0$. Second, $\left.Y_{i}=\operatorname{intflow}_{i} \underline{t}_{i}, \bar{t}_{i}\right]\left(P_{i}\right)$ for all $i \geq 0$, where $P_{i}$ is the $D_{i}$-polyhedron of $X_{i}$ and $\left[\underline{t}_{i}, \bar{t}_{i}\right] \in T_{i}$. The maximal set of abstract trajectories, the abstract semantics induced by $\left\{D_{v}\right\}$ and $\left\{T_{v}\right\}$, overapproximates the concrete semantics in the sense that every concrete trajectory (see Eq. 3) has an abstract trajectory that subsumes it, i.e., modes and switches match, $x_{i} \in X_{i}, t_{i} \in\left[\underline{t}_{i}, \bar{t}_{i}\right]$, and $y_{i} \in Y_{i}$, for all $i \geq 0$.

Computing the abstraction involves several difficulties. First, the trajectories might be not finitary. Indeed, this is unsolvable in theory, because the reachability problem is undecidable [21]. Second, the post operators are hard to compute. In particular, obtaining the sets $X$ and $Y$ in terms of conjunctions of linear inequalities in $\mathbb{R}^{n}$ requires eliminating quantifiers. In Algorithm 1 , we present a procedure (which does not necessarily terminate) for tackling the first problem. In the next section, we show how to tackle the second using support functions.

We employ Algorithm 1 to explore the tree of abstract trajectories. We store in the stack $W$ the leaves to process $\ldots(v, X)$, followed by a candidate interval $[\underline{t}, \bar{t}]$. For each leaf, we retrieve $P$, the template polyhedron of $X$. If it leads to a bad mode, we return, otherwise we search for a completeness threshold $t^{*}$ between $\underline{t}$ excluded and $\bar{t}$, checking for inclusion in the union of visited polyhedra $P_{v}$. In case of failure, we extend the time horizon of $\Delta$ and push the next candidate to the stack. Then, we partition the time between $\underline{t}$ and $t^{*}$, construct the flowpipe, and process switching. Upon each successful switch, we augment $P_{v^{\prime}}$ with the $D_{v^{\prime}}$-polyhedron of the switching region $X^{\prime}$, avoiding to store redundant polyhedra. Notably, the latter operation is efficient because all polyhedra 
comply with the same template. For the same reason, we obtain efficient inclusion checks, which we implement by first computing the template polyhedron of the left hand side, and then comparing the constant terms of the respective linear inequalities.

In conclusion, this reachability procedure that takes a template $\left\{D_{v}\right\}$ and a partitioning $\left\{T_{v}\right\}$ and constructs a tree of reachable sets of states $X$ and $Y$. It manipulates them through the post operators and overapproximate them into template polyhedra. In the next section, we discuss how to efficiently represent $X$ and $Y$, so to efficiently compute their template polyhedra. In Sect. 6 we discuss how to discover appropriate $\left\{D_{v}\right\}$ and $\left\{T_{v}\right\}$, so to eliminate spurious counterexamples.

\section{$5 \quad$ Space Abstraction Using Support Functions}

Abstracting away time left us with the task of representing the state space of the hybrid automaton, namely the space of its variable valuations. Such sets consists of polyhedra emerging from operations such as intersections, Minkowski sums, and linear maps with simple or interval matrices. In this section, we discuss how to represent precisely all sets emerging from any of these operations by means of their support functions (Sect. 5.1) and then how to abstract them into template polyhedra (Sect. 5.2). In the next section, we discuss how to refine the abstraction.

\subsection{Support Functions}

The support function of a closed convex set $X \subseteq \mathbb{R}^{n}$ in direction $d \in \mathbb{R}^{n}$ consists of the maximizer scalar product of $d$ over $X$

$$
\rho_{X}(d)=\sup \left\{d^{\top} x \mid x \in X\right\},
$$

and, indeed, uniquely represents any closed convex set [28]. Classic work on the verification of hybrid automata with affine dynamic have posed a framework for the construction of support functions from basic set operations, but under the assumption of unboundedness and nonemptiness of the represented set, and with approximated intersection [16]. Indeed, if the set is empty then its support function is $-\infty$, while if it is unbounded an $d$ points toward a direction of recession is $+\infty$, making the framework end up into undefined values. Such conditions turn out to be limiting in our context, first because we find desirable to represent unbounded sets so to accelerate the convergence to a fixpoint of the abstraction procedure, but most importantly because when encoding support functions for long abstract trajectories we might be not aware whether its concretization is infeasible. Checking this is a crucial element of a counterexample-guided abstraction refinement routine.

Recent work on the verification of hybrid automata with constant dynamics, i.e., with flows defined by constraints on the derivative only, provides us with 
a generalization of the classic support function framework which relaxes away the assumptions of boundedness and nonemptiness and yields precise intersection [7]. The framework encodes combinations of convex sets of states into LP (linear programs) which enjoy strong duality with their support function. Similarly, we encode the support function in direction $d$ of any set $X$ into the LP

$$
\begin{aligned}
& \text { minimize } c^{\top} \lambda \\
& \text { subject to } A \lambda=B d,
\end{aligned}
$$

over the nonnegative vector of variables $\lambda$. The LP is dual to $\rho_{X}(d)$, which is to say that if the LP is infeasible then $X$ is unbounded in direction $d$, and if the LP is unbounded then $X$ is the empty set. Moreover, if the LP has bounded solution so does $\rho_{X}(d)$ and the solutions coincide.

The construction is inductive on operations between sets. For the base case, we recall that from duality of linear programming the support function of a polyhedron given by a system of inequalities $P x \leq q$ is dual to the LP over $\lambda \geq 0$

$$
\begin{aligned}
& \text { minimize } q^{\top} \lambda \\
& \text { subject to } P^{\top} \lambda=d .
\end{aligned}
$$

Then, inductively, we assume that for the set $X \subseteq \mathbb{R}^{n}$ we are given an LP with the coefficients $A_{X}, B_{X}$, and $c_{X}$, and similarly for the set $Y \subseteq \mathbb{R}^{n}$. For the support functions of $X \oplus Y, M X$, and $X \cap Y$ we respectively construct the following LP over the nonnegative vectors of variables $\lambda, \mu, \alpha$, and $\beta$ :

$$
\begin{aligned}
& \text { minimize } c_{X}^{\top} \lambda+c_{Y}^{\top} \mu \\
& \text { subject to } A_{X} \lambda=B_{X} d \text { and } A_{Y} \mu=B_{Y} d, \\
& \text { minimize } c_{X}^{\top} \lambda \\
& \text { subject to } A_{X} \lambda=B_{X} M^{T} d \text {, and } \\
& \text { minimize } c_{X}^{\top} \lambda+c_{Y}^{\top} \mu \\
& \text { subject to } A_{X} \lambda-B_{X}(\alpha-\beta)=0 \text { and } \\
& \qquad A_{Y} \mu+B_{Y}(\alpha-\beta)=B_{Y} d .
\end{aligned}
$$

Such construction follows as a special case of [7], which we extend with the support function of a map through an interval matrix.

The time abstraction of Sect. 4 additionally requires us to represent the map of sets of states through interval matrices. Precisely, we are given convex set of nonnegative values $X \subseteq \mathbb{R}_{\geq 0}^{n}$, the coefficients for the respective LP, an interval matrix $[\underline{M}, \bar{M}] \subseteq \mathbb{R}^{n \times n}$, and we aim at computing the support function of all values in $X$ mapped by all matrices in $[\underline{M}, \bar{M}]$. To this end, we define the LP

$$
\begin{aligned}
\operatorname{minimize} & c_{X}^{\top} \lambda \\
\text { subject to } & A_{X} \lambda+B_{X}\left(\underline{M}^{\top} \mu-\bar{M}^{\top} \nu\right)=0 \text { and } \\
& -\mu+\nu=d,
\end{aligned}
$$

over the vectors $\lambda, \mu$, and $\nu$ of nonnegative variables. This linear program corresponds to the the dual of the interval matrix map in Eq. 8. 


\subsection{Computing Template Polyhedra}

We represent all space abstractions $X$ and $Y$ in our procedure by their support functions. In particular, whenever set operations are applied, instead of solving the operation by removing quantifiers, we construct an LP. We delay solving it until we need to compute a template polyhedron. In that case, we compute the $D$-polyhedron of the set $X$ by computing its support function in each of the directions in $D$, and constructing the intersection of halfspaces $\cap\left\{d^{\top} x \leq \rho_{X}(d) \mid\right.$ $d \in D\}$.

\section{Abstraction Refinement Using Space-Time Interpolants}

The reachability analysis of hybrid automata by means of the combination of interval arithmetic and support functions presented in Sects. 4 and 5 builds an overapproximation of the system dynamics. It is always sound for safety, but it may produce spurious counterexamples, due to an inherent lack of precision of the time abstraction and the polyhedral approximation. The level of precision is given by two factors, namely the choice of time partitioning and the choice of template directions, excluding the parameters for approximation of the exponential function, which we assume constant (see Sect. 4.1). In the following, we present a procedure to extract infeasibility proofs from spurious counterexamples. We produce them in the form of time partitions and bounding polyhedra, which we call space-time interpolants. Space-time interpolants can then be used to properly refine time partitioning and template directions.

Consider the bounded path $v_{0}, e_{0}, v_{1}, e_{1}, \ldots, v_{k}, e_{k}, v_{k+1}$ over the control graph and a sequence of dwell time intervals $\left[\underline{t}_{0}, \bar{t}_{0}\right],\left[\underline{t}_{1}, \bar{t}_{1}\right], \ldots,\left[\underline{t}_{k}, \bar{t}_{k}\right]$ emerging from an abstract trajectory. We aim at extracting a sequence $X_{0}, X_{1}, \ldots, X_{k+1}$ of (possibly nonconvex) polyhedra and a sequence $T_{0}, T_{1}, \ldots, T_{k}$ of refinements of the respective dwell times such that $Z_{0} \subseteq X_{0}$, jump juintflow $_{0}^{T_{0}}\left(X_{0}\right) \subseteq X_{1}$, $\ldots$, jump $_{k} \circ \operatorname{intflow}_{k}^{T_{k}}\left(X_{k}\right) \subseteq X_{k+1}$, and $X_{k+1} \cap I_{k+1}$ is empty. In other words, we want every $X_{i+1}$ to contain all states that can enter mode $v_{i+1}$ after dwelling on $v_{i}$ between $\underline{t}_{i}$ and $\bar{t}_{i}$ time, and the last to be separated from the invariant of mode $v_{k+1}$. Containment is to hold inductively, namely $X_{i+1}$ has to contain what is reachable from $X_{i}$, and the time refinements $T$ are to be chosen in such a way that containment holds in the abstraction. Then, we call the sequence $X_{0}, T_{0}, X_{1}, T_{1}, \ldots, X_{k}, T_{k}, X_{k+1}$ a sequence of space-time interpolants for the path and the dwell times above.

We compute a sequence of space-time interpolants by alternating multiple strategies. First, for the given sequence of dwell times, we attempt to extract a sequence of halfspace interpolants using linear programming (Sect. 6.1). In case of failure, we iteratively partition the dwell times in sets of smaller intervals, separating nonswitching from switching times and until every combination of intervals along the path admits halfspace interpolants (Sect. 6.2). We accumulate all halfspaces to form a sequence of unions of convex polyhedra that, together with the obtained time partitionings, will form a valid sequence of space-time interpolants. Finally, we refine the abstraction using the time partitionings and 
the outwards pointing directions of all computed halfspaces, in order to eliminate the spurious counterexample (Sect. 6.3).

\subsection{Halfspace Interpolation}

Halfspace interpolants are the special case of space-time interpolants where every polyhedron in the sequence is defined by a single linear inequality [1]. Indeed, they are the simplest kind of space-time interpolants, and, for the same reason, the ones that best generalize the reachable states along the path. Unfortunately, not all paths admit halfspace interpolants, but, if one such sequence exists, then it can be extrapolated from the solution of a linear program.

Consider a path $v_{0}, e_{0}, \ldots, v_{k+1}$ with the respective dwell times $\left[\underline{t}_{0}, \bar{t}_{0}\right], \ldots$, $\left[\underline{t}_{k}, \bar{t}_{k}\right]$. A sequence of halfspace interpolants consists of a sequence of sets $H_{0}, \ldots, H_{k+1}$ among either any halfspace, or the empty set, or the universe, such that $Z_{0} \subseteq H_{0}$, jump 0 o intflow $\left.\underline{\underline{t}}_{0}, \bar{t}_{0}\right]\left(H_{0}\right) \subseteq H_{1}, \ldots$, jump $\left._{k} \circ \operatorname{intflow}_{k} \underline{t}_{k}, \bar{t}_{k}\right]\left(H_{k}\right) \subseteq$ $H_{k+1}$, and $H_{k+1} \cap I_{k+1}$ is empty. In contrast with general space-time interpolants, every time partition consists of a single time interval and therefore the support function of every post operator jump $\circ$ intflow ${ }^{[\underline{t}, \bar{t}]}$ can be encoded into a single LP (see Sect. 5). We exploit the encoding for extracting halfspace interpolants, similarly to a recent interpolation technique for PCD systems [7].

We encode the support function in direction $d$ of the closure of the image of the post operators along the path, i.e., the set jump ${ }_{k} \circ$ intflow $_{k}\left[\underline{t}_{k}, \bar{t}_{k}\right] \circ \cdots \circ$ jump $_{0} \circ$ intflow $\left.\underline{t}_{0}, \bar{t}_{0}\right]\left(Z_{0}\right)$, intersected with the invariant $I_{k+1}$. We obtain the following LP over the free vectors $\alpha_{0}, \ldots, \alpha_{k+1}$ and the nonnegative vectors $\beta, \delta_{0}, \ldots, \delta_{k}$, $\gamma_{0}, \ldots, \gamma_{k+1}, \mu_{0}, \ldots, \mu_{k}$, and $\nu_{0}, \ldots, \nu_{k}$ :

$$
\begin{aligned}
& \operatorname{minimize} q_{Z_{0}}^{\top} \beta+\sum_{i=0}^{k}\left(q_{I_{i}}^{\top} \gamma_{i}+q_{G_{i}}^{\top} \delta_{i}+s_{i}^{\top} \alpha_{i+1}\right)+q_{I_{k+1}}^{\top} \gamma_{k+1} \\
& \text { subject to } P_{Z_{0}}^{\top} \beta \quad=\alpha_{0} \text {, } \\
& \underline{M}_{i}^{\top} \mu_{i}-\bar{M}_{i}^{\top} \nu_{i} \quad=-\alpha_{i} \quad \text { for each } i \in[0 . . k] \text {, } \\
& -\mu_{i}+\nu_{i}+P_{I_{i}}^{\top} \gamma_{i}+P_{G_{i}}^{\top} \delta_{i}=R_{i}^{\top} \alpha_{i+1} \quad \text { for each } i \in[0 . . k] \text {, } \\
& P_{I_{k+1}}^{\top} \gamma_{k+1}=-\alpha_{k+1}+d,
\end{aligned}
$$

where every system of inequalities $P x \leq q$ corresponds to the constraints of the respective init, guard, or invariant, every $R_{i} x+s_{i}$ is an update equation, and every interval matrix $\left[\bar{M}_{i}, \underline{M}_{i}\right]=\operatorname{intexp}\left(A_{i}, \underline{t}_{i}, \bar{t}_{i}\right)$. In general, one can check whether the closure is contained in a halfspace $a^{\top} x \leq b$ by setting the direction to its linear term $d=a$ and checking whether the objective function can equal its constant term $b$. In particular, we check for emptiness, which we pose as checking inclusion in $0 x \leq-1$. Therefore, we set $d=0$ and the objective function to equal -1 . Upon affirmative answer, from the solution $\alpha_{0}^{\star}, \alpha_{1}^{\star}, \ldots, \nu_{k}^{\star}$ we obtain a valid sequence of halfspace interpolants whose $i$-th linear term is given by $\alpha_{i}^{\star}$ and $i$-th constant term is given by $q_{Z_{0}}^{\top} \beta^{\star}+\sum_{j=0}^{i-1}\left(q_{I_{j}}^{\top} \gamma_{j}^{\star}+q_{G_{j}}^{\top} \delta_{j}^{\star}+s_{j}^{\top} \alpha_{j+1}^{\star}\right)$. 


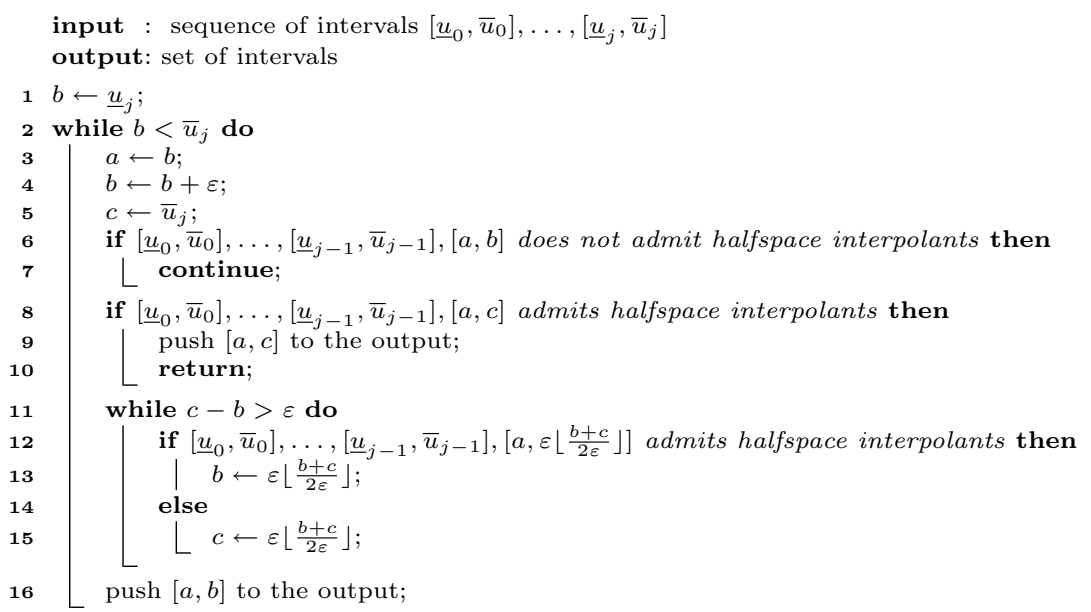

Algorithm 2. Nonswitching time partitioning.

\subsection{Time Partitioning}

Halfspace interpolation attempts to compute a sequence of enclosures that are convex for a sequence of sets that are not necessarily convex. Specifically, it requires each halfspace to enclose the set of solutions of a linear differential equation, which is nonconvex, by enclosing its convex overapproximation along a whole time interval. As a result, large time intervals produce large overapproximations, on which halfspace interpolation might be impossible. Likewise, shorter intervals produce tighter overapproximations, which are more likely to admit halfspace interpolants. In this section, we exploit such observation to enable interpolation over large time intervals. In particular, we properly partition the time into smaller subintervals and we treat each of them as a halfspace interpolation problem. Later, we combine the results to refine the abstraction.

Time partitioning is a delicate task in the whole abstraction refinement loop. In fact, while template refinement affects linearly the performance of the abstractor, partitioning time intervals that can switch induces branching in the search, possibly leading to an exponential blowup. For this reason, we partition time by narrowing down the switching time, for incremental precision, until no more is left. In particular, we use Algorithm 2 to compute a set $N$ of maximal intervals that admit halfspace interpolants, by enlarging or narrowing them of $\varepsilon$ amounts. We embed this procedure in Algorithm 3 which, along the sequence, excludes the time in $N$, constructing a set of intervals $S$ that overapproximate the switching time. In particular, we construct the set with the widest possible intervals that are disjoint from $N$. Algorithm 3 succeeds when no more intervals are left, otherwise we half $\varepsilon$ and reapply it to the sequences that are left to process. 


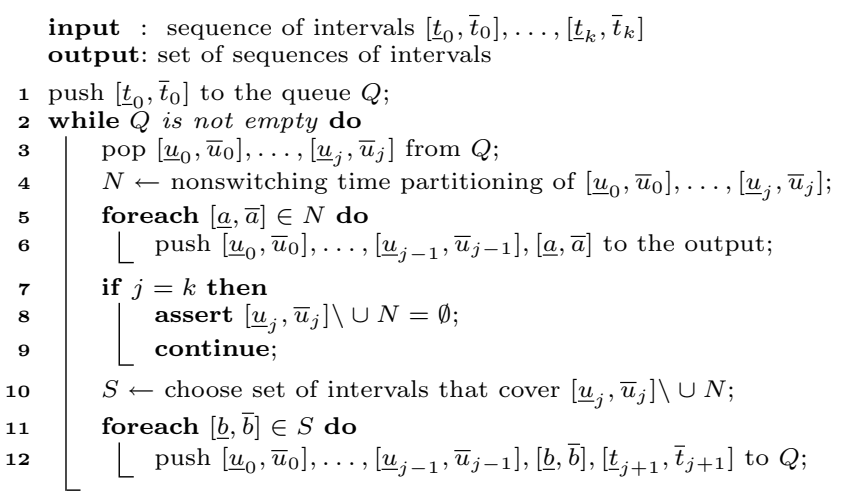

Algorithm 3. Dwell time partitioning.

\subsection{Abstraction Refinement}

The procedures above construct sequences of time intervals $\left[\underline{u}_{0}, \bar{u}_{0}\right], \ldots,\left[\underline{u}_{j}, \bar{u}_{j}\right]$ that are included in $\left[\underline{t}_{0}, \bar{t}_{0}\right], \ldots,\left[\underline{t}_{k}, \bar{t}_{k}\right]$ and that, with the respective halfspace interpolants, this constitutes a proof of infeasibility for the counterexample. Yet, it does not form a sequence of space-time interpolants $X_{0}, T_{0}, \ldots, X_{k+1}$. We form each partitioning $T_{i}$ by splitting $\left[\underline{t}_{i}, \bar{t}_{i}\right]$ in such a way each element of $T_{i}$ is either contained in $\left[\underline{u}_{i}, \bar{u}_{i}\right]$ or disjoint from it, for all intervals $\left[\underline{u}_{i}, \bar{u}_{i}\right]$. Then, we refine the partitioning of mode $v_{i}$ similarly. Each polyhedron $X_{i}$ is a union of convex polyhedra, each of which is the intersection of all halfspaces $H_{i}$ corresponding to some sequence $\left[\underline{u}_{0}, \bar{u}_{0}\right], \ldots,\left[\underline{u}_{i}, \bar{u}_{i}\right]$. Nevertheless, to refine the abstraction we do not need to construct $X_{i}$, but just to take the outward point directions of all $H_{i}$ and add them to the template of $v_{i}$.

\section{$7 \quad$ Experimental Evaluation}

We implemented our method in C++ using GMP and Eigen for multiple precision linear algebra, Arb for interval arithmetic, and PPL for linear programming $[5,23]$. In particular, all libraries we are using are meant to provide guaranteed solutions, as well as our implementation. We evaluate it on several instances of a filtered oscillator and a rod reactor, which are both parametric in the number of variables, and the latter in the number of modes too $[15,35]$. We record several statistics from every execution of our tool: the number \#cex of counterexamples found during the CEGAR loop, the number \#dir of linearly independent directions and the average width of the time partitionings extracted from all space-time interpolants. Moreover, we independently measure three times. First, the time spent in finding counterexamples, namely the total time taken by inconclusive abstractions which returned a spurious counterexample. Second, the refinement time, that is the total time consumed by computing space-time interpolants. Finally, the verification time, that is the time spend in the last 
abstraction of the CEGAR loop, which terminates with a fixpoint proving the system safe. We compare the outcome and the performance of our tool against Ariadne which, to the best of our knowledge, is the only verification tool available that is numerically sound and time-unbounded [11].

Table 1. Statistics for the benchmark examples (oot when $>1000$ s).

\begin{tabular}{l|cc|ccc|cccc|c} 
& $\begin{array}{c}\# \\
\text { vars }\end{array}$ & $\begin{array}{c}\# \\
\text { modes }\end{array}$ & $\begin{array}{c}\# \\
\text { cex }\end{array}$ & $\begin{array}{c}\# \text { dirs } \\
\text { width }\end{array}$ & $\begin{array}{c}\text { avg. } \\
\text { time }\end{array}$ & $\begin{array}{c}\text { cex. } \\
\text { time }\end{array}$ & $\begin{array}{c}\text { ver. } \\
\text { time }\end{array}$ & $\begin{array}{c}\text { tot. } \\
\text { time }\end{array}$ & Ariadne \\
\hline filtosc_1st_ord & 3 & 4 & 7 & 13 & 0.55 & 0.57 & 0.96 & 0.13 & $\mathbf{1 . 6 6}$ & 27.56 \\
filtosc_2nd_ord & 4 & 4 & 7 & 15 & 0.55 & 0.83 & 1.78 & 0.20 & $\mathbf{2 . 8 1}$ & 150.7 \\
filtosc_3rd_ord & 5 & 4 & 7 & 16 & 0.55 & 1.28 & 4.65 & 0.32 & $\mathbf{6 . 2 5}$ & oot \\
filtosc_4th_ord & 6 & 4 & 7 & 18 & 0.55 & 1.53 & 11.39 & 0.37 & $\mathbf{1 3 . 2 9}$ & oot \\
filtosc_5th_ord & 7 & 4 & 7 & 19 & 0.55 & 2.61 & 26.60 & 0.70 & $\mathbf{2 9 . 3 7}$ & - \\
filtosc_6th_ord & 8 & 4 & 7 & 18 & 0.55 & 4.56 & 101.8 & 1.29 & $\mathbf{1 0 7 . 7}$ & - \\
filtosc_7th_ord & 9 & 4 & 7 & 18 & 0.55 & 4.36 & 109.9 & 1.13 & $\mathbf{1 1 4 . 6}$ & - \\
filtosc_8th_ord & 10 & 4 & 7 & 17 & 0.55 & 5.92 & 150.9 & 1.54 & $\mathbf{1 5 8 . 4}$ & - \\
filtosc_9th_ord & 11 & 4 & 7 & 16 & 0.55 & 6.49 & 383.1 & 1.83 & $\mathbf{3 9 1 . 3}$ & - \\
filtosc_10th_ord & 12 & 4 & 7 & 17 & 0.55 & 12.84 & 428.87 & 3.73 & $\mathbf{4 4 5 . 4}$ & - \\
filtosc_11th_ord & 13 & 4 & 7 & 17 & 0.55 & 15.10 & 525.2 & 4.38 & $\mathbf{5 4 4 . 6}$ & - \\
\hline reactor_1_rod & 2 & 4 & 11 & 3 & 0.11 & 5.24 & 10.64 & 1.59 & $\mathbf{1 7 . 4 7}$ & oot \\
reactor_2_rods & 3 & 5 & 9 & 7 & 0.79 & 5.68 & 5.36 & 2.33 & $\mathbf{1 3 . 3 7}$ & oot \\
reactor_3_rods & 4 & 6 & 12 & 13 & 1.07 & 14.46 & 13.94 & 13.13 & $\mathbf{4 1 . 5 3}$ & -
\end{tabular}

The filtered oscillator is hybrid automaton with four modes that smoothens a signal $x$ into a signal $z$. It has $k+2$ variables and a system of $k+2$ affine ODE, where $k$ is the order of the filter. Table 1 shows the results, for a scaling of $k$ up to the 11-th order. The first observation is that the CEGAR loop behaves quite similarly on all scalings: number of counterexamples, number of directions, and time partitionings are almost identical. On the other hand, the computation times show a growth, particularly in the refinement phase which dominates over abstraction and verification. This suggests us that our procedure exploits efficiently the symmetries of the benchmark. In particular, time partitioning seems unaffected. What affects the performance is linear programming, whose size depends on the number of variables of the system.

The rod reactor consists of a heating reactor tank and $k$ rods each of which cools the tank for some amount of time, excluding each other. The hybrid automaton has one variable $x$ for the temperature, $k$ clock variables, one heating mode, one error mode, and $k$ cooling modes. If the temperature reaches a critical threshold and no rod can intervene, it goes into an error. For this benchmark, we start with a simple template, the interval around $x$, and we discover further directions. Table 1 highlights two fundamental differences with the previous benchmark. First, the average width grows with the model size. This is because the heating mode requires finer time partitioning than the cooling modes. The cooling modes increase with the number of rods, and so does the average width over all time partitions. Second, while with the filtered oscillator the difficulty laid at interpolation, for the rod reactor interpolation is rather easy as well as finding counterexamples. Most of the time is spent in the verification 
phase, where all fixpoint checks must be concluded, without being interrupted by a counterexample. This shows the advantage of our lazy approach, which first processes the counterexamples and finally proves the fixpoint.

Our method outperforms Ariadne on all benchmarks. On the other hand, tools like Flow* and SpaceEx can be dramatically faster [9]. For instance, they analyze filtosc_8th_ord in resp. $9.1 \mathrm{~s}$ and $0.36 \mathrm{~s}$ (time horizon of 4 and jump depth of 10). This is hardly surprising, as our method has primarily been designed to comply with soundness and time-unboundedness, and pays the price for that.

\section{Related Work}

There is a rich literature on CEGAR approaches for hybrid automata, either abstracting to a purely discrete system $[3,10,27,33,34]$ or to a hybrid automaton with simpler dynamics $[22,30]$. Both categories exploit the principle that the verification step is easier to carry out in the abstract domain. The abstraction entails a considerable loss of precision that can only be counteracted by increasing the number of abstract states. This leads to a state explosion that severely limits the applicability of such approaches. In contrast, our approach allows us to increase the precision by adding template directions, which does not increase the number of abstract states. The only case where we incur additional abstract states is when partitioning the time domain. This is a direct consequence of the nonconvexity of flowpipes of affine systems, and therefore seems to be unavoidable when using convex sets in abstractions. In [26], the abstraction consists of removing selected ODE entirely. This reduces the complexity, but does not achieve any fine-tuning between accuracy and complexity. Template reachability has been shown to be very effective in both scaling up reachability tasks to more efficient successor computations $[15,31,32]$ and achieving termination even over unbounded time horizons [12]. The drawback of templates is the lack of accuracy, which may lead to an approximation error that accumulates excessively. Efforts to dynamically refine templates have so far not scaled well for affine dynamics [14]. A single-step refinement was proposed in [4], but as was illustrated in [7], the refinement needs to be inductive in order to exclude counterexamples in a CEGAR scheme.

\section{Conclusion}

We have developed an abstraction refinement scheme that combines the efficiency and scalability of template reachability with just enough precision to exclude all detected paths to the bad states. At each iteration of the refinement loop, only one template direction is added per mode and time-step. This does not increase the number of abstract states. Additional abstract states are only introduced when required by the nonconvexity of flowpipes of affine systems, a problem that we consider unavoidable. In contrast, existing CEGAR approaches for hybrid automata tend to suffer from state explosion, since refining 
the abstraction immediately requires additional abstract states. As our experiments confirm, our approach results in templates over very low complexity and terminates with an unbounded proof of safety after a relatively small number of iterations. Further research is required to extend this work to nondeterministic and nonlinear dynamics.

Acknowledgments. We thank Luca Geretti for helping us setting up Ariadne. This research was supported in part by the Austrian Science Fund (FWF) under grants S11402-N23 (RiSE/SHiNE) and Z211-N23 (Wittgenstein Award), by the European Commission under grant 643921 (UnCoVerCPS).

\section{References}

1. Albarghouthi, A., McMillan, K.L.: Beautiful interpolants. In: Sharygina, N., Veith, H. (eds.) CAV 2013. LNCS, vol. 8044, pp. 313-329. Springer, Heidelberg (2013). https://doi.org/10.1007/978-3-642-39799-8_22

2. Althoff, M.: An introduction to CORA 2015. In: Frehse, G., Althoff, M. (eds.) ARCH14-15. 1st and 2nd International Workshop on Applied veRification for Continuous and Hybrid Systems. EPiC Series in Computer Science, vol. 34, pp. 120151. EasyChair (2015)

3. Alur, R., Dang, T., Ivančić, F.: Counterexample-guided predicate abstraction of hybrid systems. Theor. Comput. Sci. 354(2), 250-271 (2006)

4. Asarin, E., Dang, T., Maler, O., Testylier, R.: Using redundant constraints for refinement. In: Bouajjani, A., Chin, W.-N. (eds.) ATVA 2010. LNCS, vol. 6252, pp. 37-51. Springer, Heidelberg (2010). https://doi.org/10.1007/978-3-642-15643$4 \_5$

5. Bagnara, R., Hill, P.M., Zaffanella, E.: The Parma Polyhedra Library: toward a complete set of numerical abstractions for the analysis and verification of hardware and software systems. Sci. Comput. Program. 72(1-2), 3-21 (2008)

6. Benvenuti, L., Bresolin, D., Collins, P., Ferrari, A., Geretti, L., Villa, T.: Assumeguarantee verification of nonlinear hybrid systems with Ariadne. Int. J. Robust Nonlinear Control 24(4), 699-724 (2014)

7. Bogomolov, S., Frehse, G., Giacobbe, M., Henzinger, T.A.: Counterexample-guided refinement of template polyhedra. In: Legay, A., Margaria, T. (eds.) TACAS 2017. LNCS, vol. 10205, pp. 589-606. Springer, Heidelberg (2017). https://doi.org/10. 1007/978-3-662-54577-5_34

8. Chen, X., Ábrahám, E., Sankaranarayanan, S.: Taylor model flowpipe construction for non-linear hybrid systems. In: RTSS 2012, pp. 183-192 (2012)

9. Chen, X., Schupp, S., Makhlouf, I.B., Ábrahám, E., Frehse, G., Kowalewski, S.: A benchmark suite for hybrid systems reachability analysis. In: Havelund, K., Holzmann, G., Joshi, R. (eds.) NFM 2015. LNCS, vol. 9058, pp. 408-414. Springer, Cham (2015). https://doi.org/10.1007/978-3-319-17524-9_29

10. Clarke, E., Fehnker, A., Han, Z., Krogh, B., Ouaknine, J., Stursberg, O., Theobald, M.: Abstraction and counterexample-guided refinement in model checking of hybrid systems. Int. J. Found. Comput. Sci. 14(04), 583-604 (2003)

11. Collins, P., Bresolin, D., Geretti, L., Villa, T.: Computing the evolution of hybrid systems using rigorous function calculus. In: Proceedings of the 4th IFAC Conference on Analysis and Design of Hybrid Systems (ADHS12), Eindhoven, The Netherlands, pp. 284-290, June 2012 
12. Dang, T., Gawlitza, T.M.: Template-based unbounded time verification of affine hybrid automata. In: Yang, H. (ed.) APLAS 2011. LNCS, vol. 7078, pp. 34-49. Springer, Heidelberg (2011). https://doi.org/10.1007/978-3-642-25318-8_6

13. Frehse, G.: PHAVer: algorithmic verification of hybrid systems past HyTech. STTT 10(3), 263-279 (2008)

14. Frehse, G., Bogomolov, S., Greitschus, M., Strump, T., Podelski, A.: Eliminating spurious transitions in reachability with support functions. In: Proceedings of the 18th International Conference on Hybrid Systems: Computation and Control, pp. 149-158. ACM (2015)

15. Frehse, G., Le Guernic, C., Donzé, A., Cotton, S., Ray, R., Lebeltel, O., Ripado, R., Girard, A., Dang, T., Maler, O.: SpaceEx: scalable verification of hybrid systems. In: Gopalakrishnan, G., Qadeer, S. (eds.) CAV 2011. LNCS, vol. 6806, pp. 379-395. Springer, Heidelberg (2011). https://doi.org/10.1007/978-3-642-22110-1_30

16. Le Guernic, C., Girard, A.: Reachability analysis of hybrid systems using support functions. In: Bouajjani, A., Maler, O. (eds.) CAV 2009. LNCS, vol. 5643, pp. 540-554. Springer, Heidelberg (2009). https://doi.org/10.1007/978-3-642-02658$4 \_40$

17. Halbwachs, N., Proy, Y.-E., Raymond, P.: Verification of linear hybrid systems by means of convex approximations. In: Le Charlier, B. (ed.) SAS 1994. LNCS, vol. 864, pp. 223-237. Springer, Heidelberg (1994). https://doi.org/10.1007/3-54058485-4_43

18. Henzinger, T., Ho, P.H., Wong-Toi, H.: HyTech: a model checker for hybrid systems. Softw. Tools Technol. Transf. 1, 110-122 (1997)

19. Henzinger, T.A.: The theory of hybrid automata. In: Inan, M.K., Kurshan, R.P. (eds.) Verification of Digital and Hybrid Systems, vol. 170, pp. 265-292. Springer, Heidelberg (2000). https://doi.org/10.1007/978-3-642-59615-5_13

20. Henzinger, T.A., Ho, P.H., Wong-Toi, H.: Algorithmic analysis of nonlinear hybrid systems. IEEE Trans. Autom. Control 43, 540-554 (1998)

21. Henzinger, T.A., Kopke, P.W., Puri, A., Varaiya, P.: What's decidable about hybrid automata? In: Proceedings of the Twenty-Seventh Annual ACM Symposium on Theory of Computing, 29 May-1 June 1995, Las Vegas, Nevada, USA, pp. 373-382 (1995)

22. Jha, S.K., Krogh, B.H., Weimer, J.E., Clarke, E.M.: Reachability for linear hybrid automata using iterative relaxation abstraction. In: Bemporad, A., Bicchi, A., Buttazzo, G. (eds.) HSCC 2007. LNCS, vol. 4416, pp. 287-300. Springer, Heidelberg (2007). https://doi.org/10.1007/978-3-540-71493-4_24

23. Johansson, F.: Arb: efficient arbitrary-precision midpoint-radius interval arithmetic. IEEE Trans. Comput. 66, 1281-1292 (2017)

24. Kong, S., Gao, S., Chen, W., Clarke, E.: dReach: $\delta$-reachability analysis for hybrid systems. In: Baier, C., Tinelli, C. (eds.) TACAS 2015. LNCS, vol. 9035, pp. 200205. Springer, Heidelberg (2015). https://doi.org/10.1007/978-3-662-46681-0_15

25. Moler, C., Van Loan, C.: Nineteen dubious ways to compute the exponential of a matrix, twenty-five years later. SIAM Rev. 45(1), 3-49 (2003)

26. Nellen, J., Ábrahám, E., Wolters, B.: A CEGAR tool for the reachability analysis of PLC-controlled plants using hybrid automata. In: Bouabana-Tebibel, T., Rubin, S.H. (eds.) Formalisms for Reuse and Systems Integration. AISC, vol. 346, pp. 55-78. Springer, Cham (2015). https://doi.org/10.1007/978-3-319-16577-6_3

27. Ratschan, S., She, Z.: Safety verification of hybrid systems by constraint propagation-based abstraction refinement. ACM Trans. Embed. Comput. Syst. (TECS) 6(1), 8 (2007) 
28. Rockafellar, R.T.: Convex Analysis. Princeton University Press, Princeton (1970)

29. Rohn, J.: Systems of linear interval equations. Linear Algebra Appl. 126, 39-78 (1989)

30. Roohi, N., Prabhakar, P., Viswanathan, M.: Hybridization based CEGAR for hybrid automata with affine dynamics. In: Chechik, M., Raskin, J.-F. (eds.) TACAS 2016. LNCS, vol. 9636, pp. 752-769. Springer, Heidelberg (2016). https://doi.org/ 10.1007/978-3-662-49674-9_48

31. Sankaranarayanan, S., Dang, T., Ivančić, F.: Symbolic model checking of hybrid systems using template polyhedra. In: Ramakrishnan, C.R., Rehof, J. (eds.) TACAS 2008. LNCS, vol. 4963, pp. 188-202. Springer, Heidelberg (2008). https:// doi.org/10.1007/978-3-540-78800-3_14

32. Sankaranarayanan, S., Sipma, H.B., Manna, Z.: Scalable analysis of linear systems using mathematical programming. In: Cousot, R. (ed.) VMCAI 2005. LNCS, vol. 3385, pp. 25-41. Springer, Heidelberg (2005). https://doi.org/10.1007/978-3-54030579-8_2

33. Segelken, M.: Abstraction and counterexample-guided construction of $\omega$-automata for model checking of step-discrete linear hybrid models. In: Damm, W., Hermanns, H. (eds.) CAV 2007. LNCS, vol. 4590, pp. 433-448. Springer, Heidelberg (2007). https://doi.org/10.1007/978-3-540-73368-3_46

34. Sorea, M.: Lazy approximation for dense real-time systems. In: Lakhnech, Y., Yovine, S. (eds.) FORMATS/FTRTFT-2004. LNCS, vol. 3253, pp. 363-378. Springer, Heidelberg (2004). https://doi.org/10.1007/978-3-540-30206-3_25

35. Vaandrager, F.: Hybrid systems. Images of SMC Research, pp. 305-316 (1996)

Open Access This chapter is licensed under the terms of the Creative Commons Attribution 4.0 International License (http://creativecommons.org/licenses/by/4.0/), which permits use, sharing, adaptation, distribution and reproduction in any medium or format, as long as you give appropriate credit to the original author(s) and the source, provide a link to the Creative Commons license and indicate if changes were made.

The images or other third party material in this chapter are included in the chapter's Creative Commons license, unless indicated otherwise in a credit line to the material. If material is not included in the chapter's Creative Commons license and your intended use is not permitted by statutory regulation or exceeds the permitted use, you will need to obtain permission directly from the copyright holder. 